\title{
INCISIVO Y CANINO MANDIBULAR TIPO II DE VERTUCCI: REPORTE DE UN CASO
}

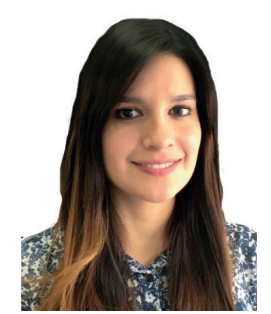

ODONT. ELIDA ORTIZ

POSGRADO ENDODONCIA UNIVERSIDAD DE CUENCA

\section{Resumen}

Este artículo presenta un reporte de caso de un incisivo y canino mandibulares tratados endodónticamente, que muestran una variación anatómica de dos conductos. Se señala la importancia del conocimiento acerca de la anatomía y sus variaciones, junto a la meticulosa observación clínica y radiográfica en busca de conductos adicionales, lo cual permite asegurar el éxito a largo plazo de la terapia endodóntica.

\section{Introducción}

Los estudios sobre la anatomía de los conductos radiculares encuentran que sus variaciones son de alta frecuencia, por lo tanto, una comprensión global de la morfología del conducto radicular y sus variaciones dictan el éxito y los resultados definitivos del tratamiento de endodoncia. (1)

El sistema de conductos en cualquier diente tiene el potencial de ser muy complejo, Vertucci clasificó las configuraciones del conducto radicular en ocho tipos de conductos radiculares:

TIPO I: Un conducto único se extiende desde la cámara al ápice.

TIPO II: Dos conductos separados parten desde la cámara pulpar y se unen próximos al ápice, terminando en uno solo.
TIPO III: Un conducto parte desde la cámara, a nivel del tercio medio se divide y posteriormente se une, terminando en un solo conducto en apical.

TIPO IV: Dos conductos separados parten de la cámara pulpar y terminan separados en apical.

TIPO V: Un conducto inicia a nivel de la cámara para luego dividirse y terminar en dos conductos separados a nivel apical .

TIPO VI: Dos conductos inician desde la cámara, a nivel del tercio medio se fusionan para luego dividirse y terminar en dos forámenes individuales.

TIPO VII: Un conducto inicia desde la cámara, a nivel medio se divide para inmediatamente 
fusionarse, y próximo al ápice nuevamente se dividen terminando de manera individual.

TIPO VIII: Tres conductos separados inician desde la cámara pulpar hasta el ápice radicular (2).

Generalmente se considera que un canino uniradicular tiene un solo conducto, pero con la investigación se ha demostrado que su anatomía es altamente variada (3).

Las raíces de los incisivos mandibulares presentan surcos longitudinales mesiales y distales que pueden conducir a la bifurcación del conducto radicular, esta puede ser parcial cuando estos dos conductos se unen y terminan en un único foramen apical, y la bifurcación total cuando los dos conductos terminan en dos forámenes apicales individuales (4). Varios estudios con diferentes etnias han demostrado que la morfología del conducto radicular varía según la raza (5). Por lo general el conducto vestibular es siempre más fácil de localizar y generalmente más recto mientras que el lingual a menudo está protegido por un hombro de dentina (6).

Radiográficamente se puede observar un estrechamiento repentino o incluso desaparece la luz del conducto, indicando que el conducto se divide en dos partes, se recomienda que las radiografías se examinen desde varios ángulos antes de acceder a la preparación (6).

\section{Reporte del caso}

Paciente de 66 años de edad, de sexo femenino fue remitida al Posgrado de Endodoncia de la Facultad de Odontología de la Universidad de Cuenca antes de la rehabilitación protésica de los dientes 4.2 y 4.3 (fig. 1)

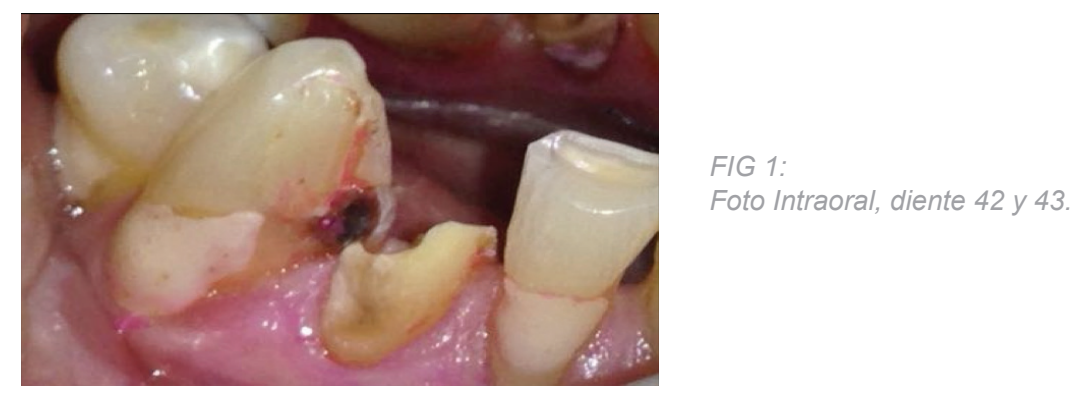

Paciente ASA I, al examen dental el diente \# 4.2 estuvo asintomático, las pruebas de sensibilidad, palpación y percusión fueron negativas. La radiografía preoperatoria no mostró ninguna zona radiolúcida, diagnosticándose como necrosis pulpar (Fig. 2A). El diente \# 4.3 estuvo asintomático, las pruebas de palpación y percusión fueron negativas. A las pruebas de sensibilidad respondió positivamente. La radiografía preoperatoria no mostró ninguna zona radiolúcida, diagnosticándose como una pulpitis irreversible asintomática (Fig.2B ).

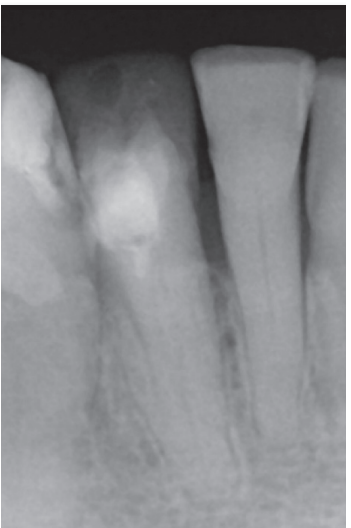

A

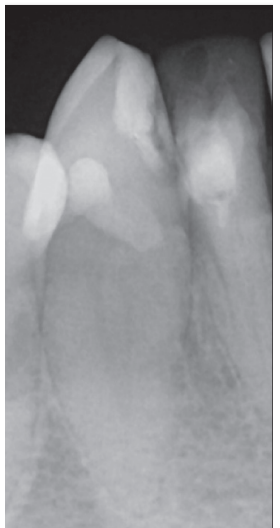

B
FIG 2:

Radiografía preoperatoria donde

se observa la bifurcación de los

conductos.

A: Diente 4.2.

B: Diente 4.3. 
Los dientes fueron tratados en citas diferentes, bajo el mismo protocolo de atención: Se administró anestesia local con Lidocaína 2\% con epinefrina 1:80.000, se aisló el diente a tratar con el dique de goma, el acceso a la cámara pulpar se logró utilizando una fresa de diamante redonda y una fresa endo-z. La exploración de la cavidad de acceso demostró la presencia de una entrada al conducto adicional, ubicado hacia lingual. Se realizó la preparación cervical con fresas Gates Glidden \# 2 y 3. (Fig. 3)

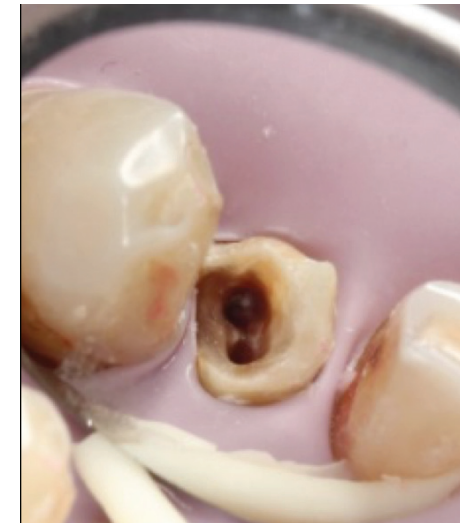

A

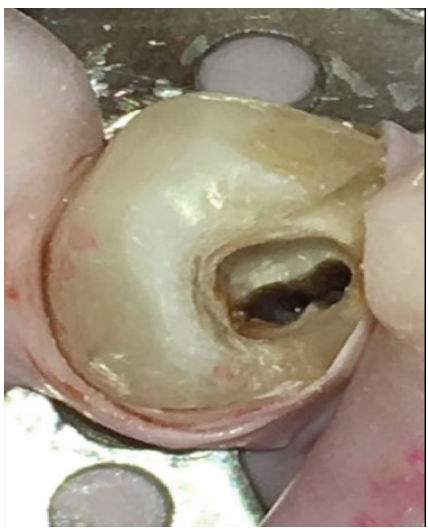

B

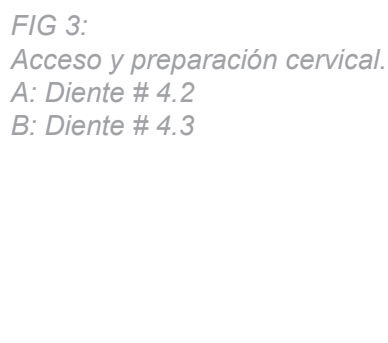

FIG 3:

yreparación cervical.

A: Diente \# 4.2

B: Diente \# 4.3

La longitud de trabajo se determinó colocando una lima K \# 15 en el conducto vestibular utilizando el localizador de ápice (Root ZX; J Morita Corp, Tokyo, Japón), de igual manera se determinó la longitud de trabajo en el conducto lingual, (LT, diente 4.2: $17 \mathrm{~mm}$ los dos conductos, diente 4.3: $26.5 \mathrm{~mm}$ los dos conductos ) se tomó la radiografía, y se observó que los conductos linguales se unen al vestibular en el tercio apical de la raíz (configuración Tipo Il de la clasificación de Vertucci) (Fig. 4).

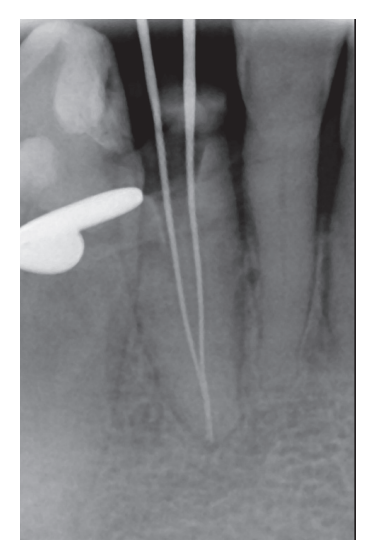

A

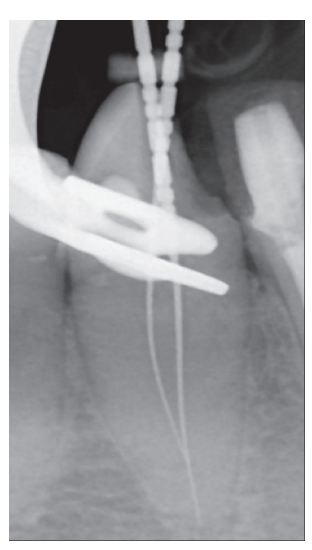

B
FIG 4:

Longitud de trabajo

A: Diente 4.2

B: Diente 4.3

Los conductos fueron limpiados y conformados con instrumentación manual usando la técnica de retroceso, la lima maestra apical fue la lima K \#35 (diente 42), lima K \# 40 (diente 4.3), durante el proceso de instrumentación se realizó patencia apical con una lima K \#10 a una longitud de $18 \mathrm{~mm}$ en el diente 4.2. Se usó hipoclorito de sodio al 5.25\% como irrigante, como parte del protocolo de irrigación final se usó EDTA al 17\% y agua destilada con activación ultrasónica.

Los conductos se secaron con puntas de papel y fueron obturados con conos de gutapercha y cemento resinoso (Topseal, Dentsply Maillefer) usando la técnica híbrida de Tagger. (Fig. 5). 


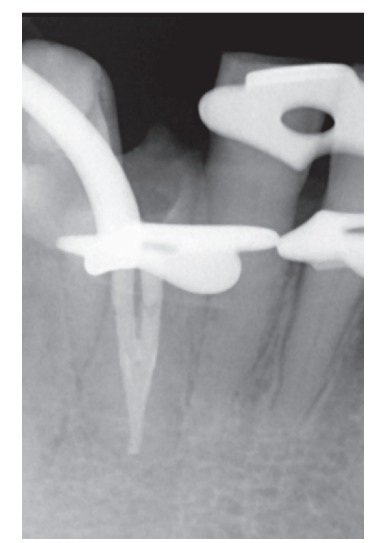

A

\section{FIG 5:}

Obturación final.

A: Diente \# 4.2

B: Diente \# 4.3

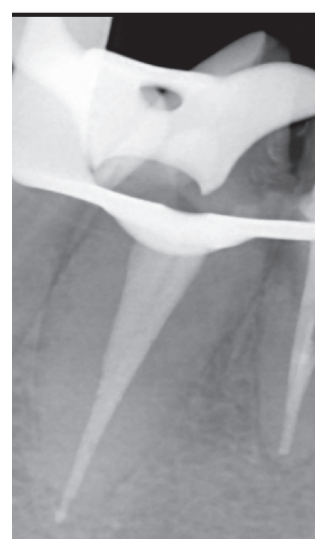

B

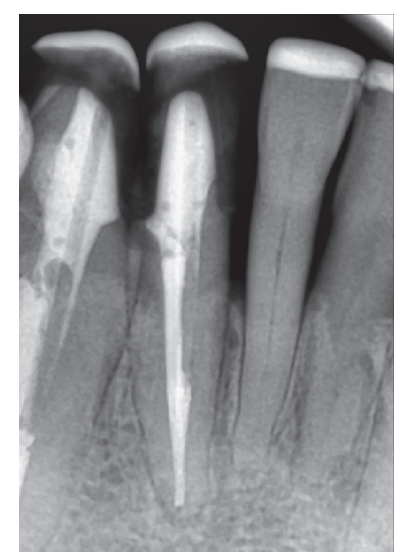

A

FIG 6:

Control a los 6 meses

A: Diente \# 4.2

B: Diente \# 4.3

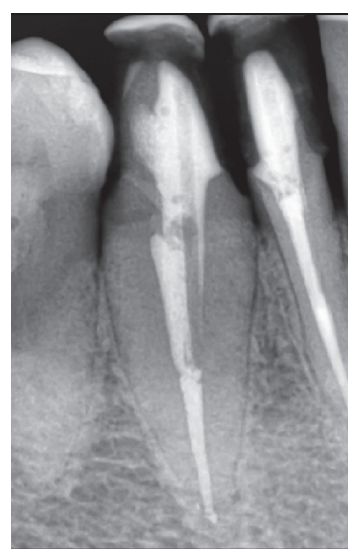

B

\section{Discusión}

Durante los últimos años, ha habido muchos estudios sobre la morfología pulpar, el conocimiento de estas variaciones es esencial para el tratamiento exitoso, existe una considerable tasa de frecuencia de la presencia de dos conductos en dientes antero inferiores, y no tratarlos es una de las principales causas del fallo endodóntico (7), debido a que el conducto no tratado puede contener tejido pulpar necrosado y de esta manera liberar subproductos nocivos (6), manteniendo o generando sintomatología dolorosa y ensanchamiento del ligamento periodontal (9).

Varios estudios coinciden con la variación anatómica descrita en este caso. Rankine-Wilson y Henry en 1965 informaron segundos conductos en un $40,5 \%$ en incisivos inferiores. Benjamin y Dowson encontraron una frecuencia de dos conductos en los incisivos inferiores en un $41.4 \%$. Por otro lado Walker en 1988 informó que la presencia del segundo conducto en los incisivos mandibulares era poco frecuente en personas de origen asiático, pero más frecuente en personas de origen europeo.

La anatomía pulpar del canino inferior es altamente variada, Aminsobhani reporta la presencia de un conducto en un $71,8 \%$ y de dos conductos en un $28,2 \%$ (8). Vertucci en 1984 , Pecora en 1993, reportaron una prevalencia de caninos inferiores tipo I en un $78 \%$ a $98 \%$ respectivamente, Versiani en el 2011 reportó caninos con dos raíces y dos conductos. Somalinga mediante tomografía de haz de cono observó de acuerdo a la clasificación de Vertucci caninos inferiores tipo I en un $79,6 \%$, tipo II 3,2\%, tipo III $13,6 \%$, tipo $\vee 2 \%(1)$.

La frecuencia del segundo conducto en los diferentes estudios están dentro de un rango similar, las variaciones en los resultados pueden deberse a diferencias en el diseño del estudio, técnica de identificación, diferencia racial, tamaño de la muestra, edad etc. (7)

La bifurcación del conducto radicular juega un papel importante en el tratamiento endodóntico, en la bifurcación parcial, la obturación de un conducto generalmente el vestibular sella- 
rá consecuentemente el extremo del otro. Sin embargo esto no ocurrirá cuando se encuentre una bifurcación total con dos forámenes apicales individuales, y la falta de tratamiento de un conducto conducirá al fracaso (7).
Una cuidadosa exploración endodóntica, así como radiografías anguladas puede conducir a la sospecha o la identificación de conductos adicionales lo cual es esencial para alcanzar el éxito terapéutico (7).

\section{Conclusión}

Este reporte de caso muestra la presencia de un segundo conducto en un incisivo lateral y canino mandibular uniradiculares. Es importante tener en consideración esta variante anatómica durante la terapia endodóntica, por lo que se recomienda ampliamente la observación cuidadosa de la cámara pulpar, así como una interpretación crítica de las radiografías y la preparación de un acceso adecuado. De esta manera se trata de concieciar a los odontólogos generales y especialistas sobre las variaciones en la morfología dental en los dientes antero-inferiores y demostrar que se necesita una mayor atención para detectar y tratar las mismas.

\section{BIBLIOGRAFÍA}

\footnotetext{
1. Somalinga Amardeep N, Raghu S, Natanasabapathy V. Root Canal Morphology of Permanent Maxillary and Mandibular Canines in Indian Population Using Cone Beam Computed Tomography. Anat Res Int. 6 de mayo de 2014;2014:e731859.

Vertucci FJ. Root canal anatomy of the human permanent teeth. Oral Surg Oral Med Oral Pathol. noviembre de 1984;58(5):589-99.

3. Versiani MA, Pécora JD, Sousa-Neto MD. Microcomputed tomography analysis of the root canal morphology of single-rooted mandibular canines. Int Endod J. septiembre de 2013;46(9):800-7.

4. Walton RE. Endodontic radiographic technics. Dent Radiogr Photogr. 1973;46(3):51-9.

5. Sert S, Bayirli GS. Evaluation of the root canal configurations of the mandibular and maxillary permanent teeth by gender in the Turkish population. J Endod. junio de 2004;30(6):391-8.

6. Muhammad A. Feroze A.Laila S. Frequency of second canal in mandibular lateral incisors (in-vitro) Pakistan Oral \& Dental Journal March 2014; 34(1)

7. Bolla N, Kavuri SR. Maxillary canine with two root canals. J Conserv Dent JCD. enero de 2011;14(1):80-2

8. Kulkarni NR, Kamat SB, Hugar SI, Nanjannawar GS. Mandibular Canine with Two Roots and Two Root Canals - A Rare Case. J Clin Diagn Res JCDR. septiembre de 2016;10(9):ZJ07-ZJ08.

9. Oliveira SHG, de Moraes LC, Faig-Leite H, Camargo SEA, Camargo CHR. In vitro incidence of root canal bifurcation in mandibular incisors by radiovisiography. J Appl Oral Sci Rev FOB. junio de 2009;17(3):234-9.
} 